\title{
Bivariate Zero-Inflated Power Series Distribution
}

\author{
Patil Maruti Krishna ${ }^{1}$, Shirke Digambar Tukaram ${ }^{2}$ \\ ${ }^{1}$ Department of Statistics, P. V. P. Mahavidyalaya, Kavathe Mahankal, Sangli, India \\ ${ }^{2}$ Department of Statistics, Shivaji University, Kolhapur, India \\ E-mail:mkpatil_stats@rediffmail.com,dtshirke@gmail.com \\ Received December 19, 2010; revised May 12, 2011; accepted May 15, 2011
}

\begin{abstract}
Many researchers have discussed zero-inflated univariate distributions. These univariate models are not suitable, for modeling events that involve different types of counts or defects. To model several types of defects, multivariate Poisson model is one of the appropriate models. This can further be modified to incorporate inflation at zero and we can have multivariate zero-inflated Poisson distribution. In the present article, we introduce a new Bivariate Zero Inflated Power Series Distribution and discuss inference related to the parameters involved in the model. We also discuss the inference related to Bivariate Zero Inflated Poisson Distribution. The model has been applied to a real life data. Extension to k-variate zero inflated power series distribution is also discussed.
\end{abstract}

Keywords: Bivariate Zero-Inflated Power Series Distribution, Bivariate Zero-Inflated Poisson Distribution, K-Variate Zero-Inflated Power Series Distribution

\section{Introduction}

In a manufacturing process there may exist several types of (say $m$ ) defects - for example, solder short circuits, solder voids, absence of solder etc. on one printed circuit board. These defects cause different types of product failure and generate different types of equipment problems. In the above example there can be only one type of defect which occurs more frequently and the other defects occurs very rarely. Another situation could be both types of defects occur rarely and so on. To model several types of defects, multivariate Poisson model is one of the appropriate models to use. This can further be modified to incorporate inflation at zero and we can have multivariate zero-inflated Poisson (MZIP) distribution. There are several ways to construct MZIP distributions. In the literature, Chin-Shang et al. [1] have discussed various types of MZIP models and investigated their distributional properties. Deshmukh and Kasture [2] have studied bivariate distribution with truncated Poisson marginal distributions. Gupta et al. [3] have considered inflated distributions at the point zero and studied the structural properties of the inflated distribution. Gupta et al. [4] have discussed score test for zero-inflated generalized Poisson regression model. Holgate [5] described the es- timation of covariance parameter of bivariate Poisson distribution by iterative method. Lambert [6] considered zero-inflated Poisson regression model. Laxminarayana et al. [7] have studied bivariate Poisson distribution and the distributional properties of the model. Patil and Shirke [8] studied testing parameter of the power series distribution of a zero-inflated power series model. Patil and Shirke [9] also studied equality of inflation parameters of two zero-inflated power series distributions. It appears that majority of the study in the literature is restricted to Poisson distribution and its extension to multivariate set up. Relatively less has been reported for the family of distributions containing other distributions.

In this article, we introduce a new Bivariate Zero-Inflated Power Series Distribution (BZIPSD) and discuss inference related to the parameters involved in the same. The rest of the paper is organized as follows. Section 2, introduces the BZIPSD along with moments of the same. Section 3, deals with inference related to the parameters involved in the BZIPSD. In Section 4, we discuss inference related to Bivariate Zero-Inflated Poisson Distribution (BZIPD). The data set reported by Arbous and Kerrich [10] is modeled by Bivariate Zero Inflated Poisson Distribution. The paper concludes with generalization to multivariate setup. 


\section{Bivariate Zero-Inflated Power Series Distribution}

Let $X$ and $Y$ be two random variables with probability mass functions

$$
\begin{aligned}
& P_{1}\left(x, \theta_{1}\right)=\frac{a(x) \theta_{1}^{x}}{f_{1}\left(\theta_{1}\right)} \text { and } P_{2}\left(y, \theta_{2}\right)=\frac{b(y) \theta_{2}^{y}}{f_{2}\left(\theta_{2}\right)} \\
& x, y \in T .
\end{aligned}
$$

where $T$ is the common support of $X$ and $Y, \theta_{1}>0$, $\theta_{2}>0, a(),. b()>.0 \quad f_{1}\left(\theta_{1}\right)=\sum a(x) \theta_{1}^{x}$, $f_{2}\left(\theta_{2}\right)=\sum b(y) \theta_{2}{ }^{y}$.
Define,

$$
\begin{aligned}
& P_{X, Y}\left(x, y, \theta_{1}, \theta_{2}, \alpha\right)=P_{1}\left(x, \theta_{1}\right) P_{2}\left(y, \theta_{2}\right) \\
& \times\left[1+\alpha\left(g_{1}(x)-E\left(g_{1}(X)\right)\right)\left(g_{2}(y)-E\left(g_{2}(Y)\right)\right)\right],
\end{aligned}
$$

where, $g_{1}(x)$ and $g_{2}(y)$ are bounded function on $\mathfrak{R}^{2}$. We note that $P_{X, Y}\left(x, y, \theta_{1}, \theta_{2}, \alpha\right)$ is a proper bivariate distribution for a suitable choice of $\alpha \in \mathfrak{R}$. Based on the distribution (2.1), in the following we introduce three types of BZIPSD.

Type-I BZIPSD: When there is an inflation only at $(x, y)^{\prime}=(0,0)^{\prime}$, we define the BZIPSD as

$$
P\left(x, y, \pi, \theta_{1}, \theta_{2}, \alpha\right)=\left\{\begin{array}{l}
(1-\pi)+\pi P_{X, Y}\left(0,0, \theta_{1}, \theta_{2}, \alpha\right), \quad(x, y)^{\prime}=(0,0)^{\prime}, 0<\pi<1 \\
\pi P_{X, Y}\left(x, y, \pi, \theta_{1}, \theta_{2}, \alpha\right), \quad(x, y)^{\prime} \neq(0,0)^{\prime}
\end{array}\right.
$$

Type-II BZIPSD: When there is inflation at $X$ component only, we define the BZIPSD as

$$
P\left(x, y, \pi, \theta_{1}, \theta_{2}, \alpha\right)=\left\{\begin{array}{l}
(1-\pi)+\pi P_{X, Y}\left(0, y, \theta_{1}, \theta_{2}, \alpha\right), \quad(x, y)^{\prime}=(0,0)^{\prime}, 0<\pi<1 \\
\pi P_{X, Y}\left(x, y, \pi, \theta_{1}, \theta_{2}, \alpha\right), \quad(x, y)^{\prime} \neq(0,0)^{\prime}
\end{array}\right.
$$

Type-III BZIPSD: When there is inflation at $Y$ - component only, we define the BZIPSD as

$$
P\left(x, y, \pi, \theta_{1}, \theta_{2}, \alpha\right)=\left\{\begin{array}{c}
(1-\pi)+\pi P_{X, Y}\left(x, 0, \theta_{1}, \theta_{2}, \alpha\right), \quad(x, y)^{\prime}=x(0,0)^{\prime}, x=0,1,2, \cdots \\
\pi P_{X, Y}\left(x, y, \pi, \theta_{1}, \theta_{2}, \alpha\right), \quad x=0,1,2, \cdots, y=1,2,3, \cdots \\
0<\pi<1
\end{array}\right.
$$

In the present discussion we focus only on Type-IBZIPSD, results on the remaining two can be obtained analogously.

\section{Moment Generating Function}

The moment generating function of $(\mathrm{X}, \mathrm{Y})$ is

$$
\begin{aligned}
& M_{X, Y}\left(t_{1}, t_{2}\right)=E\left(e^{t_{1} X+t_{2} Y}\right) \\
& M_{X, Y}\left(t_{1}, t_{2}\right) \\
& =1-\pi+\pi\left\{M_{1}\left(t_{1}\right) M_{2}\left(t_{2}\right)+\alpha\left\{\left\{E\left[e^{t_{1} X} g_{1}(X)\right]-M_{1}\left(t_{1}\right) E\left[g_{1}(X)\right]\right\}\left\{E\left[e^{t_{2} Y} g_{2}(Y)\right]-M_{2}\left(t_{2}\right) E\left[g_{2}(Y)\right]\right\}\right\}\right\}
\end{aligned}
$$

Therefore, we have

$$
\begin{aligned}
M_{X}\left(t_{1}\right) & =M_{X, Y}\left(t_{1}, 0\right) \\
& =1-\pi+\pi\left\{M_{1}\left(t_{1}\right)\right\} \\
M_{Y}\left(t_{2}\right) & =M_{X, Y}\left(0, t_{2}\right) \\
& =1-\pi+\pi\left\{M_{2}\left(t_{2}\right)\right\}
\end{aligned}
$$

where $M_{X}\left(t_{1}\right)$ and $M_{Y}\left(t_{2}\right)$ are the moment generating functions of random variables $X$ and $Y$ of zeroinflated power series distribution and $M_{1}\left(t_{1}\right)$ and $M_{2}\left(t_{2}\right)$ are the moment generating functions of random variables having power series distribution with parameters $\theta_{1}$ and $\theta_{2}$ respectively.
Suppose $f_{j}^{\prime}(\theta)$ and $f_{j}^{\prime \prime}(\theta)$ denote $\frac{\partial f_{j}(\theta)}{\partial \theta}$ and $\frac{\partial^{2} f_{j}(\theta)}{\partial \theta^{2}}$ respectively for $j=1,2$. This gives us

$$
\begin{gathered}
E(X)=M_{X}^{\prime}(0)=\frac{\pi \theta_{1} f_{1}^{\prime}\left(\theta_{1}\right)}{f_{1}\left(\theta_{1}\right)} \\
E(Y)=M_{Y}^{\prime}(0)=\frac{\pi \theta_{2} f_{2}^{\prime}\left(\theta_{2}\right)}{f_{2}\left(\theta_{2}\right)} \\
\operatorname{Var}(X)=\frac{\pi \theta_{1}}{f_{1}\left(\theta_{1}\right)}\left(\theta_{1} f_{1}^{\prime \prime}\left(\theta_{1}\right)+f_{1}^{\prime}\left(\theta_{1}\right)-\frac{\pi \theta_{1} f_{1}^{\prime}\left(\theta_{1}\right)^{2}}{f_{1}\left(\theta_{1}\right)}\right)
\end{gathered}
$$




$$
\operatorname{Var}(X)=\frac{\pi \theta_{2}}{f_{2}\left(\theta_{2}\right)}\left(\theta_{2} f_{2}^{\prime \prime}\left(\theta_{2}\right)+f_{2}^{\prime}\left(\theta_{2}\right)-\frac{\pi \theta_{2} f_{2}^{\prime}\left(\theta_{2}\right)^{2}}{f_{2}\left(\theta_{2}\right)}\right),
$$

and the correlation coefficient is

$$
\rho=\alpha \sqrt{\frac{\theta_{1} \theta_{2}}{f_{1}\left(\theta_{1}\right) f_{2}\left(\theta_{2}\right)}}\left(\frac{\left(\frac{f_{1}\left(\theta_{1} / e\right)}{e}-\frac{f_{1}\left(\theta_{1} / e\right) f_{1}^{\prime}\left(\theta_{1}\right)}{f_{1}\left(\theta_{1}\right)}\right)\left(\frac{f_{2}\left(\theta_{2} / e\right)}{e}-\frac{f_{2}\left(\theta_{2} / e\right) f_{2}^{\prime}\left(\theta_{2}\right)}{f_{2}\left(\theta_{2}\right)}\right)}{\left.\sqrt{\left(\theta_{1} f_{1}^{\prime \prime}\left(\theta_{1}\right)+f_{1}^{\prime}\left(\theta_{1}\right)-\frac{\pi \theta_{1} f_{1}^{\prime}\left(\theta_{1}\right)^{2}}{f_{1}\left(\theta_{1}\right)}\right) \sqrt{\left(\theta_{2} f_{2}^{\prime \prime}\left(\theta_{2}\right)+f_{2}^{\prime}\left(\theta_{2}\right)-\frac{\pi \theta_{2} f_{2}^{\prime}\left(\theta_{2}\right)^{2}}{f_{2}\left(\theta_{2}\right)}\right)}}\right)}\right)
$$

\section{Estimation of the Parameters of BZIPSD}

Let $\left(X_{i}, Y_{i}\right) i=1,2,3, \cdots, n$ be a random sample observed from BZIPSD $\left(\pi, \theta_{1}, \theta_{2}, \alpha\right)$. The likelihood function for the observed random sample is given by.

$$
\begin{aligned}
& L\left(\pi, \theta_{1}, \theta_{2}, \alpha ; \underline{x}, \underline{y},\right) \\
& =\prod_{i=1}^{n}\left((1-\pi)+\pi P_{X Y}\left(0,0, \theta_{1}, \theta_{2}, \alpha\right)\right)^{1-a_{i}} \\
& \times\left(\pi P_{X Y}\left(x_{i}, y_{i}, \theta_{1}, \theta_{2}, \alpha\right)\right)^{a_{i}}
\end{aligned}
$$

where $a_{i}=1$ if $\left(x_{i}, y_{i}\right) \neq(0,0)$ and $a_{i}=0$ otherwise. The corresponding log likelihood function is given by,

$$
\begin{aligned}
& \log L\left(\pi, \theta_{1}, \theta_{2}, \alpha ; \underline{x}, \underline{y},\right) \\
& =n_{0} \log \left((1-\pi)+\pi P_{X Y}\left(0,0, \theta_{1}, \theta_{2}, \alpha\right)\right) \\
& +\sum_{i=1}^{n} a_{i} \log \pi+\sum_{i=1}^{n} a_{i} \log P_{X, Y}\left(x_{i}, y_{i}, \theta_{1}, \theta_{2}, \alpha\right)
\end{aligned}
$$

$\frac{\partial \log L}{\partial \pi}=0, \frac{\partial \log L}{\partial \theta_{1}}=0, \frac{\partial \log L}{\partial \theta_{2}}=0 \quad$ and $\quad \frac{\partial \log L}{\partial \alpha}=0$ give the following equations.

$$
\begin{aligned}
& \frac{n_{0}\left(P_{X, Y}\left(0,0, \theta_{1}, \theta_{2}, \alpha\right)-1\right)}{(1-\pi)+\pi P_{X, Y}\left(0,0, \theta_{1}, \theta_{2}, \alpha\right)}+\frac{\sum_{i=1}^{n} a_{i}}{\pi}=0 \\
& \frac{n_{0} \pi P^{\left(\theta_{1}\right)}{ }_{X, Y}\left(0,0, \theta_{1}, \theta_{2}, \alpha\right)}{(1-\pi)+\pi P_{X, Y}\left(0,0, \theta_{1}, \theta_{2}, \alpha\right)} \\
& +\sum_{i=1}^{n} a_{i}\left(\frac{P_{X, Y}^{\left(\theta_{1}\right)}\left(x_{i}, y_{i}, \theta_{1}, \theta_{2}, \alpha\right)}{P_{X, Y}\left(x_{i}, y_{i}, \theta_{1}, \theta_{2}, \alpha\right)}\right)=0 \\
& \frac{n_{0} \pi P^{\left(\theta_{2}\right)}{ }_{X, Y}\left(0,0, \theta_{1}, \theta_{2}, \alpha\right)}{(1-\pi)+\pi P_{X, Y}\left(0,0, \theta_{1}, \theta_{2}, \alpha\right)} \\
& +\sum_{i=1}^{n} a_{i}\left(\frac{P^{\left(\theta_{2}\right)}{ }_{X, Y}\left(x_{i}, y_{i}, \theta_{1}, \theta_{2}, \alpha\right)}{P_{X, Y}\left(x_{i}, y_{i}, \theta_{1}, \theta_{2}, \alpha\right)}\right)=0
\end{aligned}
$$

$$
\begin{aligned}
& \frac{n_{0} \pi P^{(\alpha)}{ }_{X, Y}\left(0,0, \theta_{1}, \theta_{2}, \alpha\right)}{(1-\pi)+\pi P_{X, Y}\left(0,0, \theta_{1}, \theta_{2}, \alpha\right)} \\
& +\sum_{i=1}^{n} a_{i}\left(\frac{P^{(\alpha)}{ }_{X, Y}\left(x_{i}, y_{i}, \theta_{1}, \theta_{2}, \alpha\right)}{P_{X, Y}\left(x_{i}, y_{i}, \theta_{1}, \theta_{2}, \alpha\right)}\right)=0
\end{aligned}
$$

where $P_{X, Y}^{(\delta)}($.$) denote \frac{\partial}{\partial \delta} P_{X, Y}($.

Solving Equations (3.3) to (3.6) simultaneously we get maximum likelihood estimators of the desired four parameters. We note that all the four likelihood equations are non-linear in nature and do not have closed form solution. Now, we discuss a particular case of BZIPSD namely BZIPD.

\section{Bivariate Zero-Inflated Poisson Distribution}

Let us set $a(x)=(x !)^{-1}, b(y)=(y !)^{-1}, \quad g_{1}(x)=e^{-x}$, $g_{2}(y)=e^{-y}, f_{1}\left(\theta_{1}\right)=e^{-\theta_{1}}, f_{2}\left(\theta_{2}\right)=e^{-\theta_{2}}$ in the model (2.1). Then we get BZIPD with probability mass function.

$$
\begin{aligned}
& P(X=x, Y=y) \\
& =\left\{\begin{array}{c}
(1-\pi)+\pi e^{-\left(\theta_{1}+\theta_{2}\right)}\left(1+\alpha\left(1-e^{-\theta_{1} c}\right)\left(1-e^{-\theta_{2} c}\right)\right), \\
(x, y)^{\prime}=(0,0)^{\prime} \\
\frac{\pi e^{-\left(\theta_{1}+\theta_{2}\right)} \theta_{1}^{x} \theta_{2}^{y}}{x ! y !}\left(1+\alpha\left(e^{-x}-e^{-\theta_{1} c}\right)\left(e^{-y}-e^{-\theta_{2} c}\right)\right), \\
(x, y)^{\prime}=(0,0)^{\prime}
\end{array}\right. \\
& \text { where } c=1-(1 / e) \\
& =1-\pi+\pi \sum_{x=0}^{\infty} \sum_{y=0}^{\infty} e^{t_{1} x} P_{1}\left(x, \theta_{1}\right) e^{t_{2} y} P_{2}\left(y, \theta_{2}\right) \\
& \left(1+\alpha\left(g_{1}(x)-E\left(g_{1}(X)\right)\right) \cdot\left(g_{2}(y)-E\left(g_{2}(Y)\right)\right)\right)
\end{aligned}
$$


It is clear from the expressions of moment generating functions of $X$ and $Y$ that the marginal distributions of $X$ and $Y$ are univariate zero-inflated power series distributions with parameters $\left(\pi, \theta_{1}\right)$ and $\left(\pi, \theta_{2}\right)$ respectively. Further we have

$$
\begin{gathered}
E(X)=\pi \theta_{1} \text { and } E(Y)=\pi \theta_{2} \\
\operatorname{Var}(X)=\pi \theta_{1}\left(1+\theta_{1}(1-\pi)\right) \\
\text { and } \operatorname{Var}(Y)=\pi \theta_{2}\left(1+\theta_{2}(1-\pi)\right)
\end{gathered}
$$

The correlation coefficient is turns out to be

$$
\rho=\frac{\alpha c^{2} \sqrt{\theta_{1} \theta_{2}} e^{-\left(\theta_{1}+\theta_{2}\right) c}}{\sqrt{\left(1+(1-\pi)\left(\theta_{1}+\theta_{2}\right)+(1-\pi)^{2} \theta_{1} \theta_{2}\right)}}
$$

Remark 1: When there is no inflation $(\pi=1)$, the correlation coefficient $\rho$ is given by $\rho=\alpha c^{2} \sqrt{\theta_{1} \theta_{2}} e^{-\left(\theta_{1}+\theta_{2}\right) c}$, which coincides with the correlation coefficient given by Laxminarayan et al. [8]

Remark 2: If we choose $g($.$) to be any other suit-$ able bounded function, we will have different form of BZIPD. Some other possible functions can be $g(z)=a^{z}, \quad 0<a<1 ; \quad g(z)=e^{-z}$ etc.

Remark 3: If $\alpha=0$, we get Bivariate Zero-Inflated Poisson distribution based on two independent random variables.

\section{Estimation of the Parameters of BZIPD}

Suppose $\left(x_{i}, y_{i}\right) ; i=1,2, \cdots, n$ is a random sample observed from BZIPD $\left(\pi, \theta_{1}, \theta_{2}, \alpha\right) ; i=1,2, \cdots, n$. Then the likelihood function is given by

$$
\begin{aligned}
& L\left(\pi, \theta_{1}, \theta_{2}, \alpha ; \underline{x}, \underline{y}\right)= \\
& \prod_{i=1}^{n}\left((1-\pi)+\pi e^{-\left(\theta_{1}+\theta_{2}\right)}\left(1+\alpha\left(1-e^{-\theta_{1} c}\right)\left(1-e^{-\theta_{2} c}\right)\right)\right)^{1-a_{i}} \\
& \left(\frac{\pi e^{-\left(\theta_{1}+\theta_{2}\right)} \theta_{1}^{x_{i}} \theta_{2}^{y_{i}}}{x_{i} ! y_{i} !}\left(1+\alpha\left(e^{-x_{i}}-e^{-\theta_{1} c}\right)\left(e^{-y_{i}}-e^{-\theta_{2} c}\right)\right)\right)^{a_{i}}
\end{aligned}
$$

where $a_{i}=1$ if $\left(x_{i}, y_{i}\right) \neq(0,0)$ and $a_{i}=0$ otherwise. The corresponding log likelihood is given by,

$$
\begin{aligned}
& \log L\left(\pi, \theta_{1}, \theta_{2}, \alpha ; \underline{x}, \underline{y}\right)=n_{0} \log \left\{(1-\pi)+\pi e^{-\left(\theta_{1}+\theta_{2}\right)}\left(1+\alpha\left(1-e^{-\theta_{1} c}\right)\left(1-e^{-\theta_{2} c}\right)\right)\right\}+\sum_{i=1}^{n} a_{i} \log (\pi)-\sum_{i=1}^{n} a_{i}\left(\theta_{1}+\theta_{2}\right) \\
& +\sum_{i=1}^{n} a_{i} x_{i} \log \left(\theta_{1}\right)+\sum_{i=1}^{n} a_{i} y_{i} \log \left(\theta_{2}\right)-\sum_{i=1}^{n} a_{i} \log \left(x_{i} !\right)-\sum_{i=1}^{n} a_{i} \log \left(y_{i} !\right)+\sum_{i=1}^{n} a_{i} \log \left(1+\alpha\left(e^{-x_{i}}-e^{-\theta_{1} c}\right)\left(e^{-y_{i}}-e^{-\theta_{2} c}\right)\right)
\end{aligned}
$$

The mles of the parameters can be obtained by solving equations $\frac{\partial \log L}{\partial \pi}=0, \frac{\partial \log L}{\partial \theta_{1}}=0, \frac{\partial \log L}{\partial \theta_{2}}=0$ and

$\frac{\partial \log L}{\partial \alpha}=0$ simultaneously. These equations are given in the following:

$$
\begin{aligned}
& \frac{n_{0}\left\{-1+e^{-\left(\theta_{1}+\theta_{2}\right)}\left(1+\alpha\left(1-e^{-\theta_{1} c}\right)\left(1-e^{-\theta_{2} c}\right)\right)\right\}}{(1-\pi)+\pi e^{-\left(\theta_{1}+\theta_{2}\right)}\left(1+\alpha\left(1-e^{-\theta_{1} c}\right)\left(1-e^{-\theta_{2} c}\right)\right)}+\frac{\sum_{i=1}^{n} a_{i}}{\pi}=0 \\
& \frac{n_{0} \pi\left(e^{-\left(\theta_{1}+\theta_{2}\right)}\right)\left\{\alpha c\left(1-e^{-\theta_{2} c}\right) e^{-\theta_{1} c}-\left(1+\alpha\left(1-e^{-\theta_{1} c}\right)\left(1-e^{-\theta_{2} c}\right)\right)\right\}}{1-\pi+\pi e^{-\left(\theta_{1}+\theta_{2}\right)}\left\{1+\alpha\left(1-e^{-\theta_{1} c}\right)\left(1-e^{-\theta_{2} c}\right)\right\}} \\
& -\left(n-n_{0}\right)+\frac{\sum_{i=1}^{n} a_{i} x_{i}}{\theta_{1}}+\alpha c \sum_{i=1}^{n} a_{i}\left(\frac{e^{-\theta_{1} c}\left(e^{-y_{i}}-e^{-\theta_{2} c}\right)}{1+\alpha\left(e^{-x_{i}}-e^{-\theta_{1} c}\right)\left(e^{-y_{i}}-e^{-\theta_{2} c}\right)}\right)=0 \\
& \frac{n_{0} \pi\left(e^{-\left(\theta_{1}+\theta_{2}\right)}\right)\left\{\alpha c\left(1-e^{-\theta_{1} c}\right) e^{-\theta_{2} c}-\left(1+\alpha\left(1-e^{-\theta_{1} c}\right)\left(1-e^{-\theta_{2} c}\right)\right)\right\}}{1-\pi+\pi e^{-\left(\theta_{1}+\theta_{2}\right)}\left\{1+\alpha\left(1-e^{-\theta_{1} c}\right)\left(1-e^{-\theta_{2} c}\right)\right\}} \\
& -\left(n-n_{0}\right)+\frac{\sum_{i=1}^{n} a_{i} y_{i}}{\theta_{2}}+\alpha c \sum_{i=1}^{n} a_{i}\left(\frac{e^{-\theta_{2} c}\left(e^{-x_{i}}-e^{-\theta_{1} c}\right)}{1+\alpha\left(e^{-x_{i}}-e^{-\theta_{1} c}\right)\left(e^{-y_{i}}-e^{-\theta_{2} c}\right)}\right)=0
\end{aligned}
$$




$$
\begin{gathered}
\frac{n_{0} \pi\left(e^{-\left(\theta_{1}+\theta_{2}\right)}\right)\left\{\left(1-e^{-\theta_{1} c}\right)\left(1-e^{-\theta_{2} c}\right)\right\}}{1-\pi+\pi e^{-\left(\theta_{1}+\theta_{2}\right)}\left\{1+\alpha\left(1-e^{-\theta_{1} c}\right)\left(1-e^{-\theta_{2} c}\right)\right\}} \\
+\sum_{i=1}^{n} a_{i}\left(\frac{\left(e^{-x_{i}}-e^{-\theta_{1} c}\right)\left(e^{-y_{i}}-e^{-\theta_{2} c}\right)}{1+\alpha\left(e^{-x_{i}}-e^{-\theta_{1} c}\right)\left(e^{-y_{i}}-e^{-\theta_{2} c}\right)}\right)=0
\end{gathered}
$$

From the above equations, it is clear that Equations (4.6) to (4.9) are non-linear in nature. Solving these equations is computationally cumbersome. Laxminarayan et al. [7], adopt method of moments for the model without inflation parameter (i.e. $\pi=1$ ). In their model they have used estimates based on Method of Moment Estimators (MME), which coincide with Maximum Likelihood Estimators (MLE) of the marginal distributions. This is not the case for the joint distribution. We have to solve four equations simultaneously in order to get the MLEs. In the following we obtain maximum likelihood estimators for the following example and test for goodness of fit.

\section{An Application}

The data set in Table 1 reported by Arbous and Kerrich [10], represents accidents sustained by 122 railway men in consecutive periods of 6 and 5 years.

$X$ is the accident distribution of 122 railway men during 1937-1942 and $\mathrm{Y}$ is the accident distribution of 122 railway men during 1943-1947.

By assuming marginal distributions of $\mathrm{X}$ is ZIPD $\left(\pi, \theta_{1}\right)$. The MLEs of $X$ data are $\hat{\pi}=0.8938$ and $\hat{\theta}_{1}=1.2564$ Similarly assuming marginal distribution of $Y$ is ZIPD $\left(\pi, \theta_{2}\right)$. The MLEs of $Y$ data are $\hat{\pi}=0.8494$, $\hat{\theta}_{2}=1.3221$ Using these mles we fit the data of the mar-' ginal distribution of $X$ to ZIPD, we get Chi square statistic $=0.74843$ and $P$ value $=0.3869$. If we fit the data

Table 1. Bivariate accident distribution of 122 railway men

\begin{tabular}{|c|c|c|c|c|c|c|c|c|c|}
\hline $\mathrm{X}$ & 0 & 1 & 2 & 3 & 4 & 5 & 6 & 7 & Total \\
\hline 0 & 21 & 14 & 8 & 1 & & & & & 44 \\
\hline 1 & 17 & 12 & 8 & 3 & 1 & & & 1 & 42 \\
\hline 2 & 6 & 9 & 2 & 2 & 2 & & & & 21 \\
\hline 3 & 1 & 1 & 3 & 3 & 1 & & & & 9 \\
\hline 4 & 1 & 3 & & & & & & & 4 \\
\hline 5 & & & & 2 & & & & & 2 \\
\hline \multicolumn{10}{|l|}{6} \\
\hline \multicolumn{10}{|l|}{7} \\
\hline Total & 46 & 39 & 21 & 11 & 4 & & & 1 & 122 \\
\hline
\end{tabular}
during two periods. of the marginal distribution of $\mathrm{Y}$ to ZIPD we get Chi square statistic $=0.6065$ and $P$ value $=0.4360$. The table value of $\chi_{(1,0.05)}^{2}=3.841$. Therefore, ZIPD fits well for $X$ and $\mathrm{Y}$ data.

Thus now we can test whether data is coming from BZIPD $\left(\pi, \theta_{1}, \theta_{2}\right)$. Maximizing the log likelihood in the Equation (4.5) using MATLAB R12 software we get maximum likelihood estimators of the parameters as $\pi=0.940, \theta_{1}=1.210, \theta_{2}=1.20, \alpha=1.220$. With these parameters we fit Bivariate Zero-Inflated Poisson Distribution to the above data. The expected frequencies are as shown in the Table 2.

From the chi-square goodness of fit, we observed that calculated $\chi^{2}=4.102062$, is less than the table value of $\chi_{(4,0.05)}^{2}=9.488$. The $P$ value is 0.392369 . Hence we conclude that Bivariate Zero-Inflated Poisson Distribution fits well for the data.

Remark 4: There can be many ways to define $k$-variate ZIPSD by extending the above defined BZIPSD. One of the ways is given below.

A $k$-variate Zero-Inflated Power Series Distribution can be defined as

$$
P(\underline{x}, \pi, \underline{\theta})=\left\{\begin{array}{l}
(1-\pi)+\pi P_{X}(\underline{x}, \underline{\theta}, \alpha) \text { if } \underline{x}=\underline{0} \\
\pi P_{X}(\underline{x}, \underline{\theta}, \alpha) \text { if } \underline{x} \neq \underline{0}
\end{array}\right.
$$

where $\underline{\theta}=\left(\theta_{1}, \theta_{2}, \cdots, \theta_{k}\right)^{\prime}, \underline{X}=\left(X_{1}, X_{2}, \cdots X_{k}\right)$

$$
\begin{aligned}
& P_{X}(\underline{x}, \underline{\theta}, \alpha) \\
& =\prod_{i=1}^{k} P_{X_{i}}\left(x_{i}, \theta_{i}\right)\left(\alpha \prod_{i=1}^{k}\left\{g\left(x_{i},\right)-E\left(g\left(X_{i}\right)\right)\right\}\right)
\end{aligned}
$$

$P_{X_{i}}\left(x_{i}, \theta_{i}\right)$ is probability mass function of Power Series Distribution.

Inference related to the parameters involved in this model can be attempted similarly.

In the present work we introduced a new bivariate zero-inflated power series distribution. This distribution can accommodate number of zero-inflated bivariate discrete distributions. Further work under consideration is testing of independence for BZIPSD. Application of the

\begin{tabular}{|c|c|c|c|c|}
\hline $\mathrm{X}$ & 0 & 1 & $\geq 2$ & Total \\
\hline 0 & 21.1914 & 11.5500 & 8.7747 & 41.5161 \\
\hline 1 & 11.6746 & 15.1344 & 14.5680 & 41.3770 \\
\hline$\geq 2$ & 8.9933 & 14.7627 & 15.3421 & 39.0981 \\
\hline Total & 41.8593 & 41.4471 & 38.6848 & 121.9912 \\
\hline
\end{tabular}
proposed model for some other distributions like Bivariate Zero-Inflated Negative Binomial Distribution or k-variate zero inflated Poisson distribution can also be

Table 2. Expected frequencies using BZIPD. 
considered. These models are useful to model zero-inflated bivariate data.

\section{References}

[1] L. Chin-Shang, K. Kyungmoo, J. P. Peterson and P. A. Brinkley, "Multivariate Zero-Inflated Poisson Models and Their Applications," Technometrics, Vol. 41, No. 1, 1999, pp. 29-38. doi:10.2307/1270992

[2] S. R. Deshmukh and M. S. Kasture, "Bivariate Distribution with Truncated Poisson Marginal Distributions," Communication in Statistics: Theory and Metords, Vol. 31, No. 4, 2002, pp. 527-534. doi:10.1081/STA-120003132

[3] P. L. Gupta and R. C. Tripathi, "Inflated Modified Power Series Distributions with Applications," Communication in Statistics: Theory and Metords, Vol. 24, No. 9, 1995, pp. 2355-2374. doi:10.1080/03610929508831621

[4] R. L. Gupta and R. C. Tripathi "Score Test for ZeroInflated Generalized Poisson Regression Model," Communication in Statistics: Theory and Metords, Vol. 33, No. 1, 2004, pp. 47-64.

\section{doi: $10.1081 /$ STA-120026576}

[5] P. Holgate, "Estimation for the Bivariate Poisson Distribution," Biometrika, Vol. 51, No. 1-2, 1964, pp. 241-245.

[6] D. Lambert, "Zero-Inflated Poisson Regression, with an Application to Defects in Manufacturing," Technometrics, Vol. 34, No. 1, 1992, pp. 1-14. doi:10.2307/1269547

[7] J. Lakshiminarayana, S. N. N. Pandit and K. Srinivasa Rao, "On a Bivariate Poisson distribution," Communication in Statistics: Theory and Metords, Vol. 28, No. 2, 1999, pp. 267-276.

[8] M. K. Patil and D. T. Shirke, "Testing Parameter of the Power Series Distribution of a Zero-Inflated Power Series Model," Statistical Methodology, Vol. 4, No. 4, 2007, pp. 393-406. doi:10.1016/j.stamet.2006.12.001

[9] M. K. Patil and D. T. Shirke, "Tests for Equality of Inflation Parameters of Two Zero-Inflated Power Series Distributions," Communications in Statistics: Theory and Methods, Vol. 40, No. 14, 2011, pp. 2539-2553. doi:10.1080/03610926.2010.489172

[10] A. G. Arbous and J. E. Kerrich, "Accident Statistics and the Concept of Accident Proneness," Biometrics, Vol. 7, No. 1951, pp. 340-342. doi:10.2307/3001656 